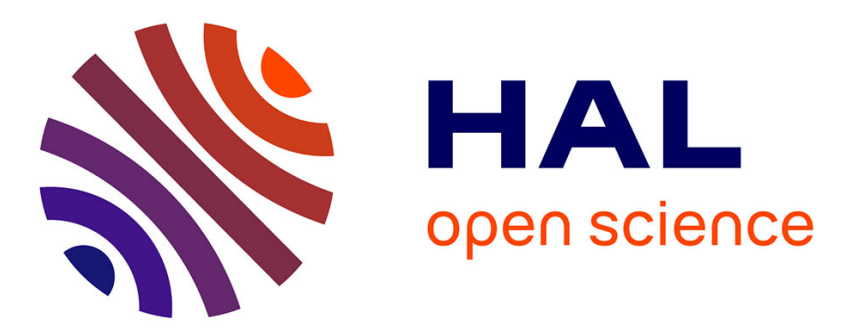

\title{
Double irradiation des molécules de bromure de méthyle et de protoxyde d'azote par rayonnements infrarouge et hertzien
}

\author{
J. Lemaire, J. Houriez, J. Thibault, Benoît Maillard
}

\section{- To cite this version:}

J. Lemaire, J. Houriez, J. Thibault, Benoît Maillard. Double irradiation des molécules de bromure de méthyle et de protoxyde d'azote par rayonnements infrarouge et hertzien. Journal de Physique, 1971, 32 (1), pp.35-40. 10.1051/jphys:0197100320103500 . jpa-00207019

\section{HAL Id: jpa-00207019 https://hal.science/jpa-00207019}

Submitted on 1 Jan 1971

HAL is a multi-disciplinary open access archive for the deposit and dissemination of scientific research documents, whether they are published or not. The documents may come from teaching and research institutions in France or abroad, or from public or private research centers.
L'archive ouverte pluridisciplinaire HAL, est destinée au dépôt et à la diffusion de documents scientifiques de niveau recherche, publiés ou non, émanant des établissements d'enseignement et de recherche français ou étrangers, des laboratoires publics ou privés. 


\title{
DOUBLE IRRADIATION DES MOLÉCULES DE BROMURE DE MÉTHYLE ET DE PROTOXYDE D'AZOTE PAR RAYONNEMENTS INFRAROUGE ET HERTZIEN
}

\author{
J. LEMAIRE, J. HOURIEZ, J. THIBAULT et B. MAILlARD \\ Faculté des Sciences, Département de Physique, \\ Laboratoire de Spectroscopie Hertzienne, B. P. 36, 59-Lille
}

(Reçu le 21 mars 1970, révisé le 2 octobre 1970)

\begin{abstract}
Résumé. - Les auteurs relatent les observations portant sur les modifications d'intensité des transitions de rotation des molécules de $\mathrm{CH}_{3} \mathrm{Br}$ et de $\mathrm{N}_{2} \mathrm{O}$ lorsque celles-ci sont soumises à un rayonnement laser. La fréquence de rayonnement infrarouge est choisie égale à la fréquence d'une transition de vibration du gaz étudié. Ils décrivent deux types différents de phénomènes, tant sur le $\mathrm{N}_{2} \mathrm{O}$ que sur le $\mathrm{CH}_{3} \mathrm{Br}$; ils les attribuent respectivement à un effet global et à un effet sélectif ; une tentative d'interprétation est proposée.
\end{abstract}

\begin{abstract}
The authors describe their observations about the modifications of the intensity of the rotational transitions of $\mathrm{CH}_{3} \mathrm{Br}$ and $\mathrm{N}_{2} \mathrm{O}$ when these molecules are submitted to laser irradiation. The frequency of the infrared radiation is chosen equal to the frequency of a rotation vibration transition of the studied gas. They describe two different types of phenomena as well on $\mathrm{N}_{2} \mathrm{O}$ as on $\mathrm{CH}_{3} \mathrm{Br}$. They attribute them respectively to a global effect and to a selective effect, and propose a tentative interpretation of the observed phenomena.
\end{abstract}

Introduction. - Dans les études de double résonance infrarouge micro-onde [1] [2], la source de rayonnement infrarouge est constituée par un laser puissant, dont les fréquences d'émission forment une suite pratiquement discrète. Aussi, ces fréquences de pompe et les fréquences de résonance du gaz ètudié ne sont généralement pas en parfaite coïncidence. Les raies d'émission laser et d'absorption du gaz possèdent un domaine de recouvrement plus ou moins important que les mesures infrarouges, trop imprécises jusqu'alors, ne permettent pas d'évaluer. Avant d'essayer d'interpréter les phénomènes observés précédemment sur la molécule de $\mathrm{CH}_{3} \mathrm{Br}$ [2], nous avons, dans une première expérience, simplifié le problème en nous assurant cette coïncidence par l'étude des transitions micro-ondes de la molécule de $\mathrm{N}_{2} \mathrm{O}$ (sonde) soumise au rayonnement infrarouge du laser à $\mathrm{N}_{2} \mathrm{O}$ (pompe).

L’appareillage utilisé précédemment [2] a été légèrement modifié (Fig. 1). Pour connaitre l'absorption du rayonnement laser par le gaz, le monochromateur suivi de son détecteur est maintenant placé à la sortie de la cellule. Une détection basée sur des techniques d'échantillonnage permet en outre l'observation des signaux en forme vraie. La fréquence micro-onde correspond au maximum d'absorption du gaz, la puissance de pompe est modulée mécaniquement par tout ou rien à la fréquence de $75 \mathrm{~Hz}$ et l'échantillonnage se fait sur les signaux de sonde contenus dans un cycle complet de $O$ à $T$, alors que la pompe est appliquée entre les instants $O$ et environ $T / 2$.

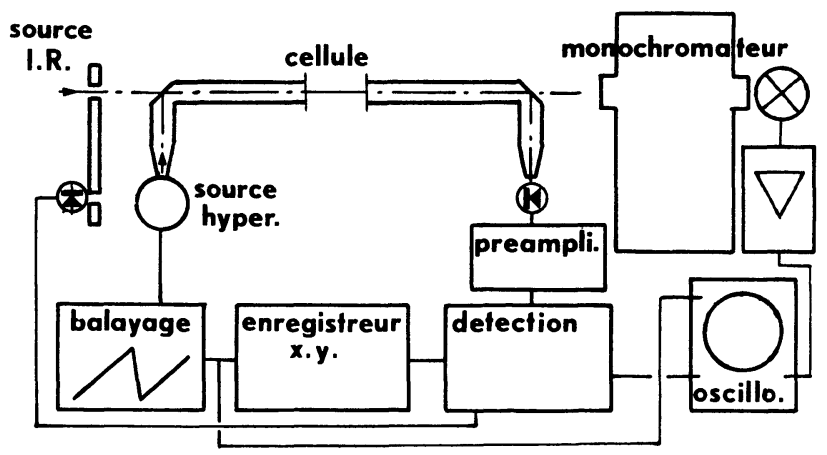

FIG. 1. - Schéma de l'appareillage

Cette technique ne fait donc apparaitre que les modifications apportées par la pompe au signal de sonde.

Etude de la molécule de $\mathrm{N}_{2} \mathrm{O}$. - La molécule de $\mathrm{N}_{2} \mathrm{O}$ est linéaire ; nous avons observé son spectre de rotation à l'aide d'un spectromètre hertzien classique de type vidéo. Parmi les transitions $J=2 \rightarrow J=3$, nous avons mesuré une raie intense à $75369,25 \mathrm{MHz}$, qui correspond à l'état fondamental de vibration [3] ; deux autres transitions correspondant à l'état de vibration dégénéré $v_{2}$ ont été pointées à $75399,26 \mathrm{MHz}$ et à $75541,70 \mathrm{MHz}$. Enfin, des techniques d'échantillonnage ont permis l'observation de deux dernières transitions de fréquence $75532,47 \mathrm{MHz}$ et $75053,40 \mathrm{MHz}$ qui correspondent respectivement aux états excités de vibration $2 v_{2}$ et $v_{1}$.

Notre étude en double irradiation s'est limitée à 


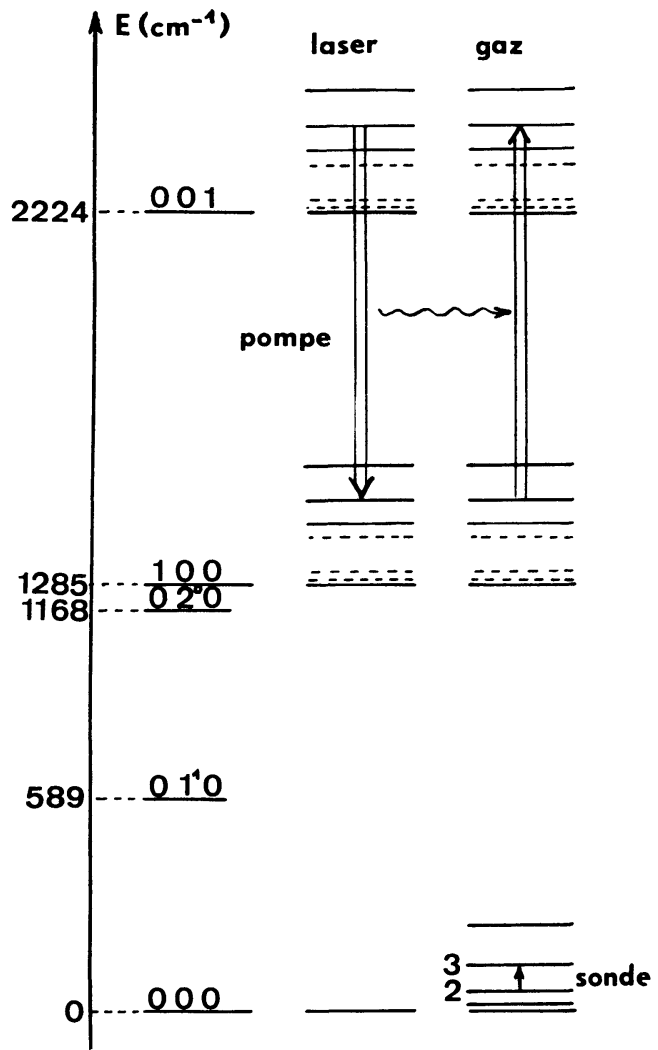

Fig. 2. - Transitions de pompe et de sonde pour la molécule de $\mathrm{N}_{2} \mathrm{O}$.

l'observation de la variation d'absorption qui correspond à la transition $J=2 \rightarrow 3$ de l'état fondamental. La sensibilité insuffisante de notre appareillage ne nous a pas encore permis d'observer des signaux sur d'autres fréquences de sonde.

La figure 2 représente les positions relatives des transitions de pompe et de sonde.
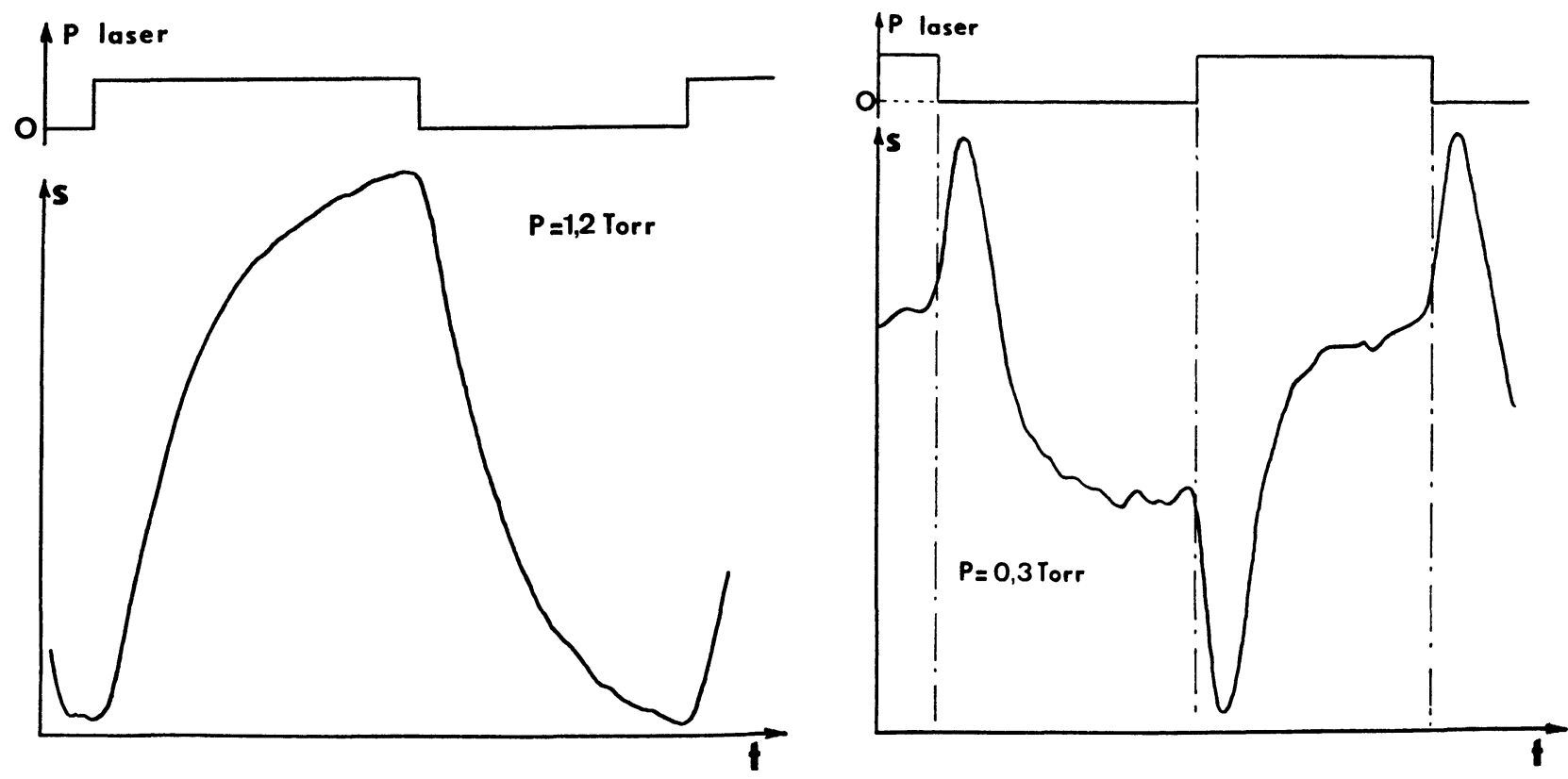

Fig. 3. - a, b, c. Evolution du signal pour la molécule de $\mathrm{N}_{2} \mathrm{O}$ en fonction de la pression. 
* La constante de temps de cette exponentielle varie dans le même sens que la pression.

* L'amplitude des signaux croît avec les puissances infrarouge et hertzienne et avec la pression.

- A faible pression ( 0,4 torr) : le signal exponentiel, qui a fortement diminué, est maintenant précédé d'un signal plus rapide, de signe opposé dont l'importance relative croît lorsque la pression diminue.

- Enfin, les signaux obtenus sur cette raie ne dépendent pas à première vue de la transition laser utilisée (branche $\mathrm{P}$ ou $\mathrm{R}$ pour $6<J<36$ ).

Etude de la molécule de $\mathbf{C H}_{3} \mathrm{Br}$. - A la suite de ces expériences, nous avons repris les études concernant la molécule de $\mathrm{CH}_{3} \mathrm{Br}$ (de l'ordre de $10^{-2}$ torr) en cherchant d'abord à déterminer la meilleure coïncidence possible entre les fréquences d'absorption de ce gaz et les raies d'émission du laser à $\mathrm{CO}_{2}$. Nous avons donc tracé pour ces différentes raies les courbes d'absorption du gaz en fonction de la pression. Nos mesures portent sur les transitions d'émission $00^{\circ} 1-10^{\circ} 0$ les plus intenses de la branche $P\left(P_{8} \ldots P_{40}\right)$. Les résuitats récapitulés ci-après ont été effectués en mesurant la pression nécessaire à l'absorption, par le gaz de la cellule, de $50 \%$ de la puissance incidente.

$\begin{array}{ccccc}\text { Transition } \ldots . & \mathbf{P}_{10} \text { et } \mathbf{P}_{38} & \mathbf{P}_{28} & \begin{array}{c}\mathbf{P}_{14}, \\ \mathbf{P}_{20} \text { et } \mathbf{P}_{22}\end{array} & \mathbf{P}_{30} \\ \text { Pression ...... } & \mathbf{0 , 1} \text { torr } & \mathbf{0 , 3} \text { torr } & 0,5 \text { torr } & 0,6 \text { torr }\end{array}$

Notons que pour les transitions $P_{10}$ et $P_{38}$, l'absorption est mesurable à très faible pression (de l'ordre de $10^{-2}$ torr) alors que cette absorption ne devient observable qu'à partir de 0,1 torr pour la transition $\mathbf{P}_{\mathbf{2 8}}$ et à partir d'une pression encore plus élevée pour les autres transitions. On peut donc penser que pour les raies $\mathbf{P}_{10}$ et $\mathbf{P}_{38}$, la coïncidence est relativement bonne. Par extrapolation des données infrarouges utilisées [4], nous pouvons formuler l'hypothèse des coïncidences de l'émission $\mathrm{P}_{10}$ de la transition $00^{\circ} 1-10^{\circ} 0 \mathrm{du} \mathrm{CO}_{2}$ avec l'absorption $J=7, K=0$ de la branche $\mathbf{P}_{\mathbf{P}}$ de la bande $v_{6}$ du $\mathrm{CH}_{3} \mathrm{Br}$ et, de l'émission $\mathrm{P}_{38}$ avec la raie $J=13, K=3$ de la même branche. Nous avons donc repris les expériences en utilisant la raie d'émission $\mathbf{P}_{10}$ plus puissante que la $\mathbf{P}_{\mathbf{3 8}}$.

Le spectre hertzien, déjà partiellement connu [5], [6], [7] ,[8], est actuellement étudié au Laboratoire où de nombreuses transitions $J=3 \rightarrow J=4$ ont été mesurées et identifiées. Ces transitions correspondent à la molécule dans son état fondamental et dans les états vibrationnels excités $v_{3}$ et $v_{6}$ dont les centres de bande se situent respectivement vers 610 et $954 \mathrm{~cm}^{-1}$.

La détection est réalisée en utilisant soit une technique d'échantillonnage, comme dans le cas de $\mathrm{N}_{2} \mathrm{O}$, soit une détection synchrone à la fréquence de modulation de la puissance laser. Cette dernière technique permet alors un balayage lent de la fréquence de sonde. Enfin, la puissance du laser à $\mathrm{CO}_{2}$ utilisé est beaucoup plus grande que celle du laser à $\mathrm{N}_{2} \mathrm{O}$.
Les résultats peuvent se diviser en 3 parties :

Détection par échantillonnage. - Lorsque la fréquence de sonde est centrée sur le maximum d'absorption des transitions les plus intenses, les signaux sont semblables à ceux obtenus pour $\mathrm{N}_{2} \mathrm{O}$ à forte pression. Ces signaux qui ont toujours la même forme exponentielle suivent les mêmes lois d'évolution en fonction de la pression et de la puissance des rayonnements. Il semble, d'après les observations effectuées sur plusieurs raies que l'amplitude des signaux soit proportionnelle à l'absorption hertzienne correspondante. Cette amplitude est dans ce cas bien supérieure à celle observée sur $\mathrm{N}_{2} \mathrm{O}$. De ce fait, nous avons pu diminuer la pression jusqu'à 0,03 torr et observer ainsi des signaux à constante de temps très faible (50 à $100 \mu \mathrm{s})$.

Détection synchrone. - Certaines régions du spectre (Fig. 4) sont explorées par balayage lent de la fréquence de sonde.

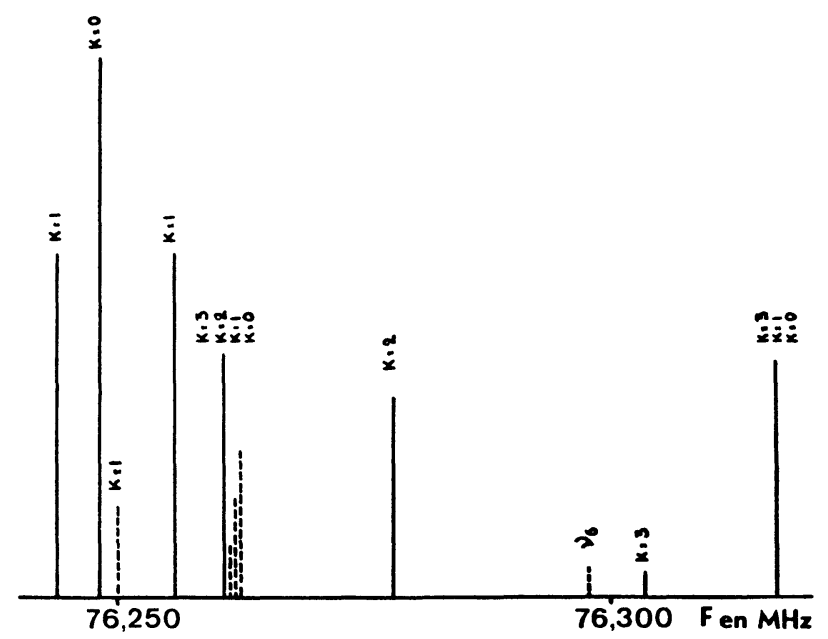

FIG. 4. - Partie du spectre où se trouvent théoriquement les transitions $J=3 \rightarrow 4$ dans l'état $v_{6}$ du $\mathrm{CH}_{3} \mathrm{Br}^{79}$.

Les raies sont séparées (la pression est de l'ordre de 0,03 torr) et donnent un signal correspondant à une diminution de l'absorption lorsqu'elles appartiennent à l'état fondamental de vibration et, un signal de sens contraire lorsqu'elles appartiennent aux niveaux $v_{3}$ ou $v_{6}$; notons que ces signaux sont du même ordre de grandeur pour ces deux états. La figure 5 représente l'enregistrement obtenu sur la partie du spectre (Fig. 4) où s'intercalent des transitions dans l'état fondamental du $\mathrm{CH}_{3} \mathrm{Br}^{81}$ et des transitions dans l'état excité $v_{6} \mathrm{du} \mathrm{CH}_{3} \mathrm{Br}^{79}$ qui n'ont pas encore été identifiées.

Détection par échantillonnage. - Le signal qui correspond aux deux transitions $3_{0} \rightarrow 4_{0}$,

$$
F=7 / 2 \rightarrow 9 / 2 \text { et } F=9 / 2 \rightarrow 11 / 2
$$

de l'état fondamental du $\mathrm{CH}_{3} \mathrm{Br}^{81}$ (76 248,49 MHz), comporte comme pour le $\mathrm{N}_{2} \mathrm{O}$ à faible pression, une partie négative avant la montée exponentielle. 


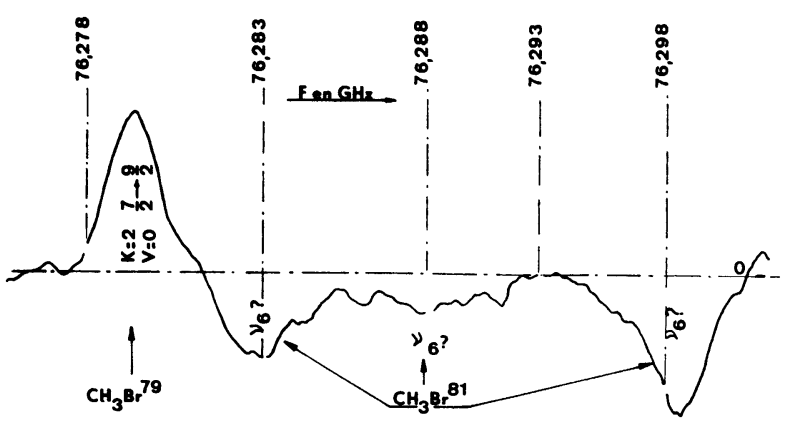

Fig. 5. - Observation de quelques transitions $J=3 \rightarrow 4$ du spectre de $\mathrm{CH}_{3} \mathrm{Br}$ par détection synchrone à la fréquence de modulation de la puissance laser.

Avec le matériel utilisé, cette partie du signal n'apparaît qu'à très faible pression ; on obtient un optimum pour une pression de l'ordre de 0,025 torr. La figure 6 montre la forme de ces signaux analysés au voisinage de la mise en route de la pompe (environ de $n T-T / 20$ à $n T+T / 20)$. Notons que le temps de montée de ces signaux (de l'ordre de $50 \mu \mathrm{s}$ ) semble être limité uniquement par la bande passante de notre appareillage. Ceci est caractéristique de cette raie, car nous n'avons rien observé de semblable pour les autres transitions $J=3 \rightarrow 4$ les plus intenses.

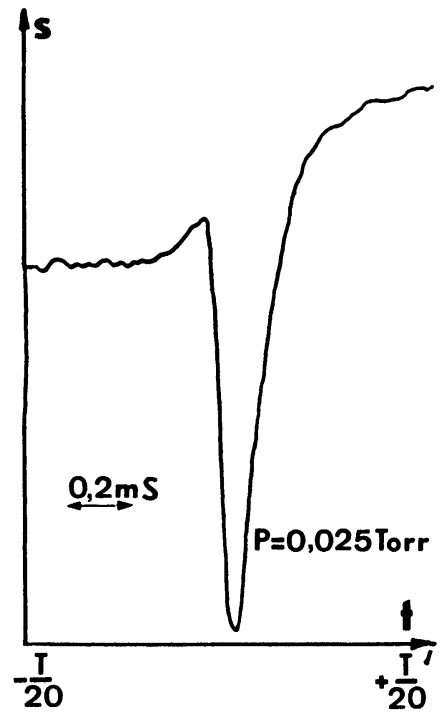

Fig. 6. - Pompage sélectif sur les transitions $J=3 \rightarrow 4$, $K=0 \rightarrow 0, F=7 / 2 \rightarrow 9 / 2$ et $F=9 / 2 \rightarrow 11 / 2$ de l'état fondamental du $\mathrm{CH}_{3} \mathrm{Br}^{81}$.

DisCUSSION DES RÉSULTATS. - L'ensemble des manipulations sur la molécule de $\mathrm{CH}_{3} \mathrm{Br}$ peut se résumer en 2 phénomènes :

- phénomène à effet non sélectif du rayonnement infrarouge, ment.

- phénomène à effet sélectif de ce même rayonne-

Le premier peut s'expliquer par une augmentation de la température globale du gaz. Les exponentielles représentent alors le passage d'un état d'équilibre à la température ambiante $T$ à un autre état d'équilibre (en présence de rayonnement infrarouge) : état qui correspond à une autre température globale $T^{\prime}>T$.

Cette hypothèse explique en particulier (voir appendice) :

- l'augmentation du signal en fonction de la puissance infrarouge,

- le sens de la variation de la constante de temps des exponentielles en fonction de la pression,

- pour chaque état de vibration, l'apparente proportionnalité entre l'amplitude des signaux et l'amplitude des raies d'absorption correspondantes,

- la présence d'un signal de signe contraire pour les transitions des états $v_{3}$ et $v_{6}$.

Le phénomène à effet sélectif comporte une partie négative qui correspond donc à une augmentation de l'absorption. La répartition en $K$ plutôt qu'en $J$ des niveaux d'énergie de la molécule de $\mathrm{CH}_{3} \mathrm{Br}$ (Fig. 7) et la règle de sélection $\Delta K=0$ suggèrent l'hypothèse d'un couplage entre le niveau de départ de la pompe $(J=7, K=0)$ et le niveau $J=4, K=0$ plus rapide qu'entre ce niveau pompé et le niveau $J=3, K=0$.

Ce couplage entre les différents niveaux $K=0$ serait alors caractérisé par une constante de temps très faible comparée à celle relative aux signaux non sélectifs.

Ces hypothèses nous permettent de tenter une interprétation des phénomènes observés avec $\mathrm{N}_{2} \mathrm{O}$. La partie des signaux de forme exponentielle peut être expliquée de la même façon que pour le $\mathrm{CH}_{3} \mathrm{Br}$, c'est-à-dire par le passage d'un état d'équilibre global à un autre. Le rapport en puissance entre le laser à $\mathrm{CO}_{2}$ et celui à $\mathrm{N}_{2} \mathrm{O}$ expliquant la différence entre les domaines de pression utilisés et entre les amplitudes des signaux observés. Il n'en est pas de même pour la partie brève du signal qui, comparée à l'information relative à l'effet sélectif sur $\mathrm{CH}_{3} \mathrm{Br}$, présente un temps de montée beaucoup moins rapide malgré une pression de gaz plus élevée.

L'interprétation de cette partie brève ne peut être basée sur l'hypothèse envisagée pour $\mathrm{CH}_{3} \mathrm{Br}$. La réaction sur le niveau fondamental du pompage infrarouge des molécules de l'état $v_{1}$ vers l'état $v_{3}$, est certainement liée à des phénomènes plus complexes.

Conclusion. - Des études systématiques, plus fines, doivent nous permettre de connaître plus précisément ces processus de couplage entre les niveaux. En particulier, il est probable qu'en complétant nos observations effectuées sur les niveaux de départ par une étude malheureusement plus difficile au voisinage des niveaux d'arrivée de la pompe, nous disposerions d'éléments nouveaux.

Pour le $\mathrm{N}_{2} \mathrm{O}$, les niveaux de départ sont situés vers $1285 \mathrm{~cm}^{-1}$ et ceux d'arrivée vers $2224 \mathrm{~cm}^{-1}$; l'intensité des raies directement couplées avec la transition de sonde est donc faible et leur observation difficile. De plus, les émissions laser ne commencent à être puissantes que pour des valeurs de $\mathbf{J}$ supérieures 


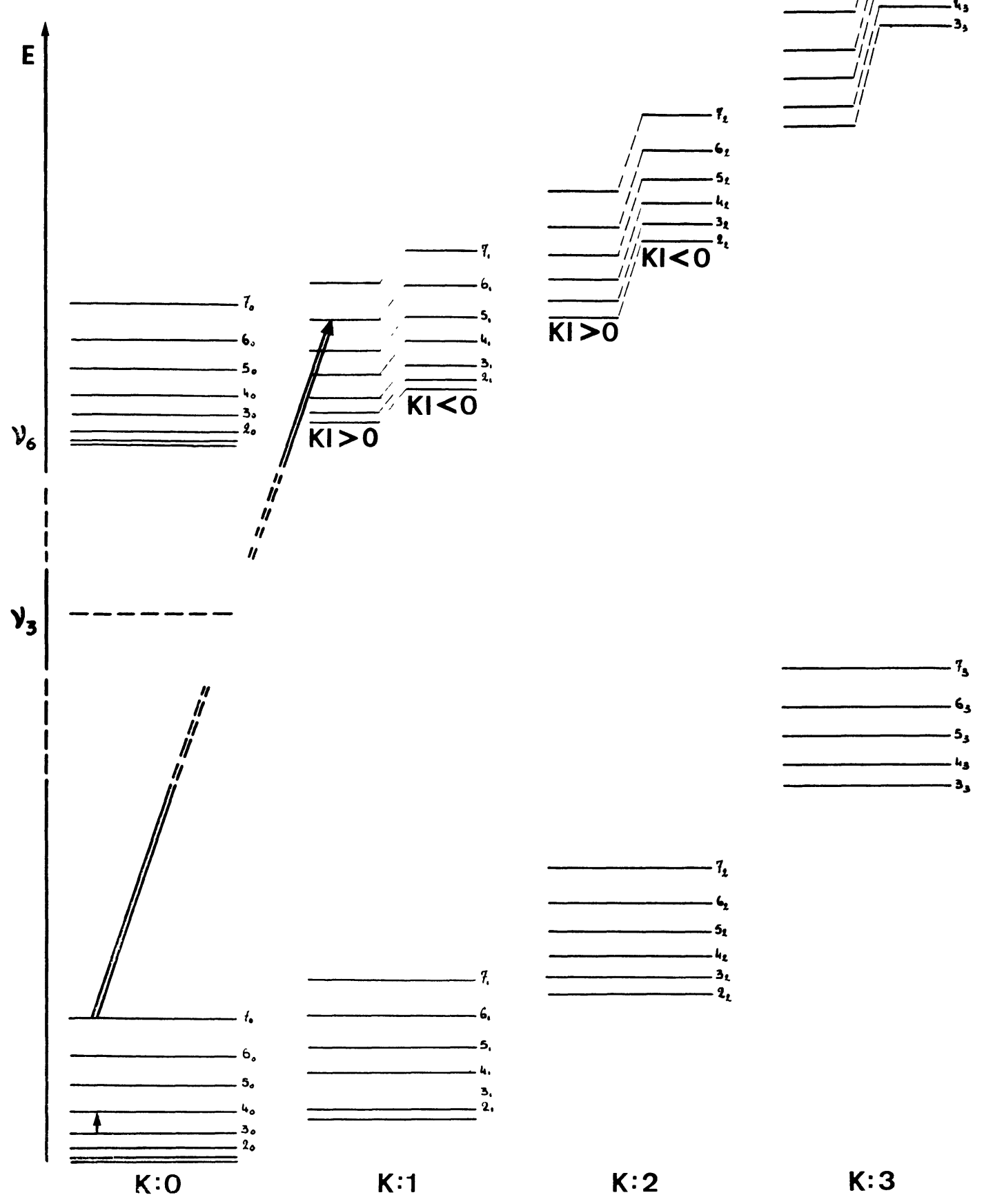

FIG. 7. - Répartition des niveaux d'énergie pour la molécule de $\mathrm{CH}_{3} \mathrm{Br}$ et représentation de la transition de sonde et de la transition de pompe (selon notre hypothèse).

à 10 , les transitions hertziennes correspondantes se situent vers $250 \mathrm{GHz}$ et leur observation nécessite des techniques micro-ondes plus délicates. Pour le $\mathrm{CH}_{3} \mathrm{Br}$, les 2 transitions hertziennes directement couplées au niveau de départ $J=7$ se situeraient selon notre hypothèse vers $133 \mathrm{GHz}$ et $152 \mathrm{GHz}$, celles du niveau d'arrivée vers 114 et $133 \mathrm{GHz}$, leur étude est donc moins délicate que dans le cas $\mathrm{N}_{2} \mathrm{O}$.

Appendice. - Dans l'hypothèse d'un effet non sélectif, il est possible de déterminer l'équation d'échauffement du gaz. En se limitant au cas d'une enceinte cylindrique de rayon $a$ dont tout le volume est irradié, on obtient une équation différentielle de la forme :

$$
p+\chi\left(\frac{1}{r} \frac{\partial \theta}{\partial r}+\frac{\partial^{2} \theta}{\partial r^{2}}\right)=\rho c \frac{\partial \theta}{\partial t}
$$

ou $p=$ puissance absorbée par unité de volume,

$\chi=$ conductibilité thermique du gaz,

$\rho=$ masse volumique,

$c=$ capacité calorifique,

$\theta=\theta(r, t):$ différence entre la température du gaz et la température initiale. 
En admettant que le gaz au voisinage de l'enceinte est à la température des parois supposée constante, on peut déterminer $\theta(r, t)$ par la méthode de Fourier.

$$
\begin{aligned}
\theta(r, t) & =\frac{p a^{2}}{4 \chi} \times \\
& \times\left[1-\left(\frac{r}{a}\right)^{2}-8 \sum_{n=1}^{\infty} \frac{J_{0}\left(\gamma_{n} \frac{r}{a}\right)}{\gamma_{n}^{3} J_{1}\left(\gamma_{n}\right)} \exp \left(-\frac{\gamma_{n}^{2} \chi t}{\rho c a^{2}}\right)\right]
\end{aligned}
$$

où $\gamma_{n}$ est le zéro de rang $n$ de la fonction de Bessel d'ordre zéro.

En ne tenant compte que du premier terme de la série, on peut évaluer la variation du signal correspondant

$$
\Delta S=\int_{0}^{a} \int_{0}^{2 \pi} \frac{\alpha}{\pi a^{2}}[\theta(r, t)] r \mathrm{~d} r \mathrm{~d} \varphi
$$

$\alpha$ étant un coefficient qui représente la variation du signal $\partial S / \partial \theta$ par unité de surface de la section du guide d'onde :

$$
\Delta S=\frac{p a^{3} \alpha}{16 \chi}\left[1-\frac{32 \exp \left(-\frac{\chi \gamma_{1}^{2} t}{\rho c a^{2}}\right)}{\gamma_{1}^{5} J_{1}\left(\gamma_{1}\right)}\right]
$$

L'exposant $\left(-\chi \gamma_{1}^{2} / \rho c a^{2}\right)$ peut être mesuré expérimentalement et la théorie cinétique des gaz permet de calculer $\chi / \rho c, a$ est pris égal au rayon du cylindre de même section que le guide utilisé.

Nous nous limitons au cas de $\mathrm{N}_{2} \mathrm{O}$ car nous connaissons approximativement le diamètre de cette molécule et le domaine de pression utilisé est tel que les chocs intermoléculaires sont nettement prédominants.

Le calcul donne $\exp (-570 t / P)$ où $P$ est exprimé en Torr, alors que les mesures varient de $\exp (-600 t / P)$ (obtenu sur la Fig. $3 a)$ à $\exp (-300 t / P)$ (pour d'autres pressions). Le calcul précédent effectué à l'aide d'un modèle d'enceinte à plateaux parallèles donne

$$
\exp \left(-\frac{\pi^{2} \chi t}{4 \rho c a^{2}}\right)=\exp \left(-\frac{600 t}{P}\right)
$$

où $2 a$ est la distance entre les plateaux (prise égale au petit côté du guide).

$\mathrm{Si}$ on considère l'approximation du modèle proposé par rapport au modèle réel, les erreurs sur les mesures de pression et la validité des résultats de la théorie cinétique des gaz, on peut admettre que ces résultats théoriques et expérimentaux sont en bon accord, ce qui justifie les hypothèses proposées.

\section{Bibliographie}

[1] Ronn (A. M.) and Lide (D. R. Jr), J. Chem. Phys., 1967, 47, 3669.

[2] Lemaire (J.), Houriez (J.), Bellet (J.) et Thibault (J.), C. R. Acad. Sci. Paris, 1969, 268, 922.

[3] LAfFerTy (W. J.) and Lide Jr (D. R.), Journal of Molecular Spectroscopy, 1964, 14, 407.

[4] Jones (W. E.), PoppleWell (R. J. L.) and Thompson (H. W.), Spectrochimica Acta., 1966, 22, 647.
[5] Gordy (W.), Simmons (J. W.) and Smith (A. G.), Phys. Rev., 1948, 74, 243.

[6] Simmons (J. W.) and Anderson (W. E.), Phys. Rev., $1950,80,338$.

[7] Orville Thomas (W. J.), Cox (J. T.) and Gordy (W.), J. of chem. Phys., 1954, 22, 1718.

[8] Morino (Y.) and Hirose (C.), J. of Molecular Spectroscopy, 1967, 24, 204. 